\title{
SURFACES IN 3-MANIFOLDS: GROUP ACTIONS ON SURFACE BUNDLES
}

\author{
By IAIN R. Aitchison
}

\begin{abstract}
We discuss briefly some conjectures concerning surfaces in 3-manifolds, and describe two results on finite group actions on surface bundles over the circle.
\end{abstract}

\section{Manifolds of dimension 1 and 2}

The classification of closed orientable surfaces can be achieved by studying embedded circles on surfaces. Analogously, approaches to the classification of closed orientable 3 dimensional manifolds involve studying embedded circles and surfaces. We review these approaches, often illustrating more general results by their statements in the closed, orientable context: unless otherwise clear, manifolds considered will be closed (compact with empty boundary), connected, and orientable. Manifolds and maps are smooth. References are necessarily incomplete.

Any closed connected 1-manifold is diffeomorphic to the circle $S^{1}$. In dimension 2, either genus or Euler characteristic determine a closed orientable surface, as does the fundamental group. Any closed, orientable surface $S$ can be decomposed as a connectsum $S \cong \#_{k} T^{2}=\#_{k} S^{1} \times S^{1}$ of $k$ tori, for a unique $k \geqq 0$. (We denote by $M \# N$ the connect sum of two $n$-manifolds $M$ and $N$, obtained by removing an open ball from each, and identifying the two resulting boundary spheres.)

There are 3 classes of surface, according to whether the Euler characteristic is positive, zero, or negative. These correspond to the sphere $S^{2}$, the torus $S^{1} \times S^{1}$, and surfaces of higher genus; these classes correspond to the 3 types of homogeneous 2-dimensional geometry. Representatives admit metrics of constant curvature $+1,0$ and -1 respectively. Moreover, fundamental groups are respectively finite (in fact, trivial), infinite abelian (polynomial growth), and infinite non-abelian (exponential growth). Surface types play different but important roles in the study of 3-dimensional manifolds. As motivation, we consider common approaches to classifying a surface $S$. These usually involve cutting $S$ along $\pi_{1}$-injectıve circles: these are circles representing non-trivial homotopy classes. Very importantly, if a surface contains an immersed $\pi_{1}$-injective circle, it contains an embedded $\pi_{1}$-injective circle.

One approach is to seek an embedded circle $\gamma$ which is non-trivial homologically on $S$, and which is thus non-separatıng. Another embedded circle $\gamma^{\prime}$ meeting $\gamma$ transversely at one point can easily be found. Each of these circles has an annular neighbourhood on the surface, chosen so that their union is a torus with a disc removed. The boundary of

1991 subject classification (Amer. Math. Soc.): 57N10, 57M35, 57M50.

Received October 28, 1993. 
this punctured torus in $S$ separates $S$ into two components ( $S$ being their connect-sum), one of which having homology of strictly lower rank than that of $S$. In this approach it is necessary to show that a surface $S$ with $H_{1}(S, Z)=0$ is a sphere, or equivalently, that a homologically trivial surface with boundary a circle is necessarily a disc. Summarizing, any closed surface $S$ can be cut open along a disjoint family of non-separating circles to obtain a 2-sphere with an even number of open discs removed. This in turn can be cut open along disjointly embedded arcs to yield a disc.

The classification of surfaces can also be achieved by consecutively cutting a given surface into simpler pieces, using disjointly embedded non-parallel separating $\pi_{1}$-injective circles. Each of these determines a splitting $S=S_{1} \# S_{2}$, with each summand having homology of lower rank.

Suppose $M$ is an $n$-dimensional manifold with boundary $\partial M \neq \emptyset$. Given an embedded sphere $S^{k-1} \subset \partial M$ with neighbourhood $N=S^{k-1} \times B^{n-k}$, we can attach a $k$-handle to $M$ to form a new manifold $M^{\prime}$. This is a quotient space of the disjoint union of $M$ and $B^{n}$, where we identify a standardly embedded $S^{k-1} \times B^{n-k}$ in $\partial B^{n}$ with $N \subset \partial M$ by some diffeomorphism. With this notion, the classification of surfaces leads to the following statements:

THEOREM 1. 1. Every closed orientable 2-manifold contaıns embedded disjoint circles whose complement embeds in the 2-sphere.

2. Every closed orientable 2-manifold contains an embedded 1-manifold whose complement consists of two copies of a (planar) surface obtained by attaching 1handles to a 2-disc.

3. Every closed orientable surface occurs as the boundary of a 3-manifold (called a handlebody) obtained by attaching 1-handles to a 3-ball.

4. Every closed orientable surface occurs as a 2-fold branched cover of the 2-sphere.

5. Every closed orientable surface which is neither a 2-sphere nor a torus has infinite fundamental group, and admits a complete Riemannian metric of constant curvature -1 .

\section{3-manifolds: general considerations}

In the 1950's Moise [Moi] proved that every topological 3-manifold $M$ admits an essentially unique precewrse-linear structure (that is, can be nicely triangulated), and that such a PL-structure gives rise to a smooth structure in an essentially unique way. It is easy to see that every element $\gamma \in \pi_{1}(M ; *)$, can be represented by an embedded circle, and every class $\beta \in H_{2}(M ; Z)$ can be represented by a (possibly disconnected) embedded surface. There is the following analogue of the previous result for surfaces:

THEOREM 2. 1. Every closed orientable 3-manifold contains embedded disjoint circles whose complement embeds in the 3-sphere.

2. Every closed orientable 3-manifold contains a Heegaard surface, whose complement consısts of two copies of a handlebody obtained by attaching 1-handles to a 3-ball. 
3. Every closed orientable 3-manifold occurs as the boundary of a 4-manifold obtained by attaching 2-handles to a 4-ball.

4. Every closed orientable 3-manifold occurs as a 3-fold branched cover of the 3sphere.

5. (Conjecture) A closed orientable 3-manifold which has no non-trivial 2-spheres or torı, and has infinıte fundamental group, admits a complete Rıemannian metric of constant curvature -1 . (See below.)

Part 1 says that every 3 -manifold arises by surgery on a link $L \subset S^{3}$. This result is due independently to Lickorish and Wallace [Li,Wall]. Lickorish's proof uses Heegaard surfaces (arising from the 1-skeleton and dual 1-skeleton of a triangulated 3-manifold); he deduces the first and third. Wallace begins with the fact that every closed orientable 3 -manifold is the boundary of an orientable 4-manifold, and modifies such a description.

Unfortunately, given a surgery description of two 3-manifolds, or a Heegaard surface description, no general effectıve algorithm is known to determine whether or not the manifolds are homeomorphic. Even if they are homeomorphic, although there exists a finite list of known moves generating a transformation of one description to the other, there is no effectıve algorithm to describe an actual sequence of such moves.

Traditional approaches to understanding 3-manifold topology sought to emulate approaches to the classification of surfaces, using homotopically non-trivial surfaces to cut open a 3-manifold. But whereas there is only one connected closed 1-manifold, the three different types of surfaces each play different roles.

There are two additional complicating problems. The first is to identify 'trivial' building blocks. The second is to find surfaces along which to cut. Concerning the first, Poincare originally conjectured that a closed 3-manifold with the same integral homology as the 3-sphere is homeomorphic to the 3-sphere. After discovering a homology 3 -sphere with non-trivial fundamental group, Poincaré reformulated his conjecture to

Conjecture 3 (Poincaré). Every simply-connected homology 3 -sphere $\Sigma^{3}$ is homeomorphic to the 3 -sphere $S^{3}$.

This is still unresolved, remaining one of the most famous problems of contemporary mathematics. By Whitehead's theorem, $\Sigma^{3}$ is actually homotopy equivalent to the 3sphere; we can ask a more general question. For closed 3-manifold, when does homotopy equivalence imply homeomorphy?

\section{2-spheres in 3-manifold topology}

For any 3-manifold $M, M \# S^{3}$ is homeomorphic to $M$ : if there exists a counterexample $\Sigma^{3}$ to the Poincaré Conjecture, we do not expect $M \# \Sigma^{3}$ to be homeomorphic to $M=M \# S^{3}$. We can fortunately exploit the existence of embedded, 'non-trivial' 2-spheres to sidestep the Poincaré Conjecture as an obstacle to a successful study of 3-manifolds, using the notion of prime and ırreducible 3-manifolds.

An embedded 2-sphere $S$ in $M$ is called essential or incompressible if it separates $M$, with neither complementary component being a 3-ball, or if it is non-separating. In the latter case, an argument similar to the 2-dimensional case shows that $M=M^{\prime} \# S^{2} \times S^{1}$. 
We call $M$ ırreducible if there are no essential 2-spheres, and prıme if there are no essential separatıng 2-spheres.

If $\pi_{2}(M) \neq 0$, there exists an immersed non-trivial 2-sphere. That there then exists an embedded 2-sphere representing a non-trivial element of $\pi_{2}(M)$ is Papakyriakopolous' celebrated Sphere Theorem $[\mathrm{Pa}] .\left(S^{2}\right.$ is the only surface for which homotopic nontriviality of an immersion implies the existence of a non-trivial embedded surface.) If this sphere separates $M$, we obtain $M \cong M_{1} \# M_{2}$, where neither factor is a homotopy 3 -sphere.

THEOREM 4. Every closed, orientable 3-manifold $M$ has a (unıque) decomposition as a connect-sum $M \cong M_{1} \# M_{2} \# \ldots \# M_{k}$ of prime 3-manifolds $M_{\imath}$.

Existence was proved by Kneser [Kn], uniqueness by Milnor [Miln] (up to ordering). The fundamental group of a non-trivial connect-sum correspondingly decomposes as a free product of groups. The study of closed, orientable 3-manifolds reduces to the study of prime manifolds.

A prime 3-manifold has either finite or infinite fundamental group. In the former case, the universal cover is a homotopy 3 -sphere, and we are in the realm of the Poincare Conjecture, and problems concerning the existence of non-standard actions of finite groups on homotopy 3-spheres (that is, inequivalent to linear actions). The main examples of 3-manifolds with finite fundamental group are lens spaces. These all have Heegaard surfaces of genus 1: they contain a separating, embedded torus, which bound $S^{1} \times D^{2}$ on each side. Lens spaces are not determined up to homeomorphy by their homotopy type, but are determined by their simple homotopy type.

A prime 3-manifold with infinite fundamental group is either $S^{2} \times S^{1}$, with universal cover $S^{2} \times R^{1}$, or is irreducible. An irreducible 3-manifold with infinite fundamental group is aspherical, and has universal cover homotopy equivalent to $R^{3}$. There are uncountably many mutually non-homeomorphic contractible 3-manifolds (see [He1]), but only $R^{3}$ is known to cover a compact 3-manifold. The Poincaré Conjecture can now be extended to

Conjecture 5 (Extended Poincaré Conjecture). The unıversal cover of a prime 3-manifold is homeomorphic to one of $S^{3}, R^{3}$ or $S^{2} \times R^{1}$.

ConjeCture (Homotopy equivalence inplies homeomorphy). Two homotopy equıvalent, prime, aspherical, closed 3-manifolds are homeomorphic.

In the aspherical case, homotopy type is determined by the fundamental group: to give an effective classification of aspherical prime 3-manifold types is equivalent to giving an effective classification of their fundamental groups. For $n \geqq 4$, classification of closed $n$-dimensional manifolds is impossible: every possible group can occur, but the isomorphism problem for these groups is unsolvable (see [Ma]).

\section{Higher-genus surfaces: Haken manifolds}

In the following, all 3-manifolds considered are irreducible and aspherical. An immersion $f: S \longrightarrow M$ of a surface into a 3 -manifold is said to be $\pi_{1}$-injectıve if the 
induced map $f_{*}: \pi_{1}(S) \longrightarrow \pi_{1}(M)$ is 1:1. A $\pi_{1}$-injective embedded orientable surface, other than the 2-sphere, is called an incompressible surface in $M$. Heegaard surfaces are not $\pi_{1}$-injective, and have not proved useful in the understanding of the relationship between homotopy type and homeomorphy.

If an irreducible 3-manifold $M$ contains an incompressible surface (other than a sphere), it is said to be a Haken manifold, and non-Haken otherwise. Generally, it is not possible to produce an embedded $\pi_{1}$-injective surface from an $\imath$ mmersed one: non-Haken 3 -manifolds exist containing immersed $\pi_{1}$-injective surfaces.

A non-Haken manifold must be a rational homology 3-sphere, since infinite $\mathrm{H}_{2}\left(\mathrm{M}^{3}\right.$; $Z$ ) guarantees that $M^{3}$ contains a $\pi_{1}$-injective non-separating surface: this can only be a 2-sphere if $M^{3}$ is $S^{1} \times S^{2}$.

For some time it has been thought that a 'generic' rational homology sphere should be non-Haken: Thurston has produced many such examples by surgery on knots and links, all but finitely many appropriate surgeries yielding non-Haken manifolds. However, Hatcher has recently given an argument suggesting that most may be Haken [Hat].

An incompressible surface gives an initial surface along which to begin cutting open a manifold $M$, to obtain a 'simpler' manifold with incompressible boundary components. Finitely many further cuts (along a 'hierarchy' of $\pi_{1}$-injective surfaces) decompose $M$ into recognizable pieces. This technique enabled Waldhausen [Wa] to prove

THEOREM 7. Homotopy equıvalent Haken manifolds are homeomorphıc.

\section{Toroidal and atoroidal manifolds}

The role of tori in Haken 3-manifolds is now well-understood. If $M^{3}$ contains no $\pi_{1}$-injective torus, we call $M^{3}$ atoroıdal.

Any $Z \oplus Z$ subgroup of $\pi_{1}(M)$ can be realized as the image of the fundamental group of an immersed torus: an atoroidal 3-manifold has fundamental group containing no $Z \oplus Z$ subgroup. An embedded torus in an atoroidal, irreducible 3-manifold must bound a handlebody of genus 1 . For manifolds with boundary, an immersed torus is said to be peripheral if it can be homotoped into the boundary. Thurston [Th1,Th2,Th3] has proved

THEOREM 8. A closed Haken manifold is atoroudal if and only if it admits a metric of constant curvature -1. If an irreducible manifold with boundary has only peripheral tort (if any), then it admits a metric of constant curvature -1 .

A foliation of $M$ by circles is called a Seifert-fiberıng. Seifert-fibered manifolds (see [JS]) also admit canonical metrics [Th1,Sc]. Thurston has constructed many non-Haken 3 -manifolds admitting metrics of constant curvature -1 [Th1].

Conjecture 9 (Thurston's Geometrization Conjecture). An irreducible, atoroıdal 3-manifold with infinite $\pi_{1}$ admits a hyperbolic structure. Every closed, irreducible 3manifold is a canonical union of pieces, each admitting geometry based on one of the 8 possible homogeneous geometries. 
This includes the Poincare Conjecture. For toroidal Haken manifolds, we have the decomposition theorem of Jaco-Shalen and Johannson [JS,Jo]:

THEOREM 10. A closed Haken 3-manifold contains a maximal collection of nonparallel incompressible tori, unique up to isotopy. Complementary components are either Seifert fibered, or have only peripheral tori.

Thus the Geometrization Conjecture is true in the Haken case. The simplest examples of torus decompositions arise from the Thurston/Nielson classification of surface diffeomorpisms [Th5]. If $h: S \longrightarrow S$ is a homeomorphism, the mappıng torus of $h$ is the closed 3-manifold $S \times{ }_{h} S^{1} \equiv S \times[0,1] /(x, 0) \sim(h(x), 1)$ obtained by glueing together the two ends of a product of $S$ with the interval using $h$. We call this a surface bundle over $S^{1}$, with fiber $S$ and monodromy $h$. Any homeomorphism $h^{\prime}$ of a closed surface $S$ is isotopic to a homeomorphism $h$ preserving setwise a unique maximal collection $C$ of embedded, non-parallel circles. Moreover, some power $g=h^{k}$ of $h$ preserves the complementary components of $C$, and $g$ restricted to each is either periodic or pseudo-Anosov. The invariant circles $C$ give rise to incompressible tori, and periodic homeomorphisms give rise to Seifert-fibered pieces of $S \times{ }_{h} S^{1}$. A surface bundle admits a complete hyperbolic metric if and only if it is atoroidal and has pseudo-Anosov monodromy [Th3].

Recent work of Scott, Mess, Casson and Gabai [Sc2,Me,Ca,Ga2] shows that if a nonHaken irreducible 3 -manifold $M$ contains a $\pi_{1}$-injective torus, then $M$ is Seifert-fibered.

\section{Conjectures on surfaces in 3-manifolds}

CONJECTURE 11. Every 3-manifold with infinite fundamental group contains a $\pi_{1}$ injective surface.

If there exists such a surface (other than $S^{2}$ ) satisfying the 4-plane, 1-line condition of Hass and Scott [HS], then homotopy equivalence with $M$ implies homeomorphy. Many examples of such surfaces are given in [AR1,AR2,ALR] and the survey [AR3]. This conjecture is weaker than

CONJECTURE 12. Every non-Haken manifold is virtually Haken, that is, has a finite-sheeted Haken cover.

Hempel [He1] shows this for manifolds with orientation-reversing involutions. See also Millson [Mill]. For hyperbolic 3-manifolds, Long [Lo] proved the conjecture true if an immersed, totally geodesic surface exists. A resolution of this conjecture would be a major advance toward resolving the Geometrization Conjecture.

CONJECTURE 13. Every non-Haken manifold has a finate-sheeted Haken cover with non-zero first Betti number.

If an atoroidal, irreducible 3-manifold $M$ has non-zero second Betti number, the Thurston norm becomes a useful tool [Th4]. This is a norm on $H_{2}(M, R) \cong R^{k}$ derived from assigning to each lattice point in $Z^{k} \cong H_{2}(M, Z)$ a positive number corresponding 
to the minimal possible Euler characteristic for an embedded representative surface.

The simplest examples of such Haken manifolds are surface bundles over $S^{1}$; the fibre represents a non-trivial homology class. Stallings [St] made the first major study of surface bundles. Every 3-manifold is almost a bundle: Gonzáles-Acuña and Myers [GA,My] have shown that every 3-manifold can be obtained by surgery on a knot in a bundle over $S^{1}$. Sakuma [Sa] has proved that every closed, orientable 3-manifold has a 2 -fold branched cover which is a surface bundle.

ConjeCture 14 (Thurston). Every atoroadal, irreducible closed 3-manifold M has a finite-sheeted cover which fibres over the circle.

A discussion of this conjecture is given by Gabai [Ga]. Luecke [Lu] has proved that if an irreducible 3-manifold has an incompressible torus, then it is either finitely covered by a torus bundle over $S^{1}$, or has finite covers of arbitrarily large first Betti number. Seifert-fibered spaces are completely understood (see [Ga]).

If $M$ is hyperbolic, there are two kinds of possible immersed surfaces: geometrically finite surfaces, which are either Fuchsian (totally geodesic), or quasi-Fuchsian, and geometrically infinite surfaces. These two types correspond to the surface subgroup acting on hyperbolic space having limit set in the 2-sphere at $\infty$ either a topological circle (quasi-circle), or the whole sphere. Fibres of hyperbolic bundles correspond to geometrically infinite surfaces. An immersed geometrically infinite surface is called a virtual fibre. Thurston has proved that if $M$ contains a virtual fibre, it has a finite bundle cover to which the virtual fibre lifts as an actual fibre. Virtual fibres have been further studied by Soma [So1,So2]. Reid [Re] gave the first example - a 10-fold irregular covering by a genus 2 bundle - of a non-Haken hyperbolic 3-manifold finitely covered by a bundle.

CONJECTURE 15. Every non-Haken manifold has finate-sheeted covers with arbitrarily large first Bettı number.

\section{Surface bundles: two results}

We state two results, and sketch their proofs. The simplest coverings are regular (Galois) coverings. The simplest hyperbolic bundles are those of genus 2 . If the preceding conjectures are correct, it is natural to seek non-Haken 3-manifolds arising as the quotient of a bundle by a finite group action. The only group known to act with non-bundle quotients is $Z_{2}$ (see [Ga]). The author has some unpublished examples of other cyclic group actions with rational homology sphere quotients.

The isometry group of a hyperbolic 3-manifold $M$ is finite. Kojima [Ko] has shown that every finite group acts as the full group of isometries of some hyperbolic 3-manifold. We offer

THEOREM 16. Given a finıte group $G$, there exısts infinitely many hyperbolic 3manifolds $M$, each fibering over the curcle, on which $G$ acts freely by isometries.

The proof of the theorem is a simple application of a construction due to Penner [Pen]. Suppose we are given an embedded circle $\gamma$ on a surface S. A Dehn twist $D_{\gamma}$ 
on $\gamma$ is a diffeomorphism of $S$ defined as the identity in the complement of an annular neighbourhood $N \cong S^{1} \times[0,1]$ of $\gamma$, and on $N$ by the map $\left(e^{\theta i}, t\right) \mapsto\left(e^{(\theta+\epsilon 2 \pi t) i}, t\right), \epsilon=$ \pm 1 . It is a right twist when $\epsilon=1$, left otherwise. Up to isotopy, any diffeomorphism of an orientable surface is generated by a finite sequence of Dehn twists. Choose two families $F_{l}, F_{r}$ of finitely many disjointly embedded circles on $S$, so that all regions in the complement of $F_{l} \cup F_{r}$ are hexagons. Let $D_{l}$ and $D_{r}$ denote the diffeomorphisms respectively obtained by Dehn twisting to the right along all circles in $F_{r}$, and to the left along all circles in $F_{l}$. A diffeomorphism $\phi_{\omega}$ defined by a word $\omega$ in $D_{l}$ and $D_{r}$, containing both non-trivially, is isotopic to a pseudo-Anosov map. The mapping torus $S \times{ }_{\phi_{\omega}} S^{1}$ then admits a metric of constant curvature -1 .

The finite group $G$ acts freely on a Cayley graph for $G$, and so by equivariantly replacing vertices by 3 -balls, and edges by 1 -handles, we obtain a free action of $G$ on a handlebody $H$, and on its boundary $\partial H$. Let $S$ denote the quotient surface $\partial H / G$, and $\pi$ the covering projection. Choose two families $F_{l}, F_{r}$ as above, and for each circle $\gamma$, let $n_{\gamma}$ denote the least common multiple of all degrees of $\pi$ restricted to $\pi^{-1}(\gamma)$. A power $D_{\gamma}^{k}$ lifts to a diffeomorphism of $\partial H$ if and only if $n_{\gamma}$ divides $k$. There are many choices for a pseudo-Anosov map $\phi: S \longrightarrow S$ lifting to $\tilde{\phi}: \partial H \longrightarrow \partial H$. The covering transformation group $G$ then acts as a group of isometries on the hyperbolic manifold $\partial H \times_{\tilde{\phi}} S^{1}$

The next result concerns unıqueness of fibrations of bundles. If the first Betti number of a bundle $M$ is 1 , the fibres $F_{1}=\pi_{1}^{-1}$ (point), $F_{2}=\pi_{2}^{-1}$ (point) of two fibrations $\pi_{1}, \pi_{2}: M \longrightarrow S^{1}$ are isotopic. Any toroidal bundle has non-isotopic fibres of the same genus (by 'Dehn twisting' along tori). Thurston has proved that if the first Betti number of an atoroidal bundle $M$ is $\geqq 2$, fibres exist of arbitrarily large genus ([Th4]). This follows since the unit ball in the Thurston norm is a finite-sided compact polyhedron, and lattice points corresponding to fibrations lie on rays through the interior of certain faces. Every other lattice point on a ray through such a face also corresponds to a fibration.

THEOREM 17. 'Most' genus 2 hyperbolic bundles have a unique fibration of genus 2. The quotient of any such bundle under a regular covering projection is Haken.

Generators for diffeomorphisms of a genus 2 surface can be chosen as Dehn twists on a collection of circles, each invariant under a hyperelliptic involution. Thus every genus 2 bundle $M$ admits a hyperelliptic involution $h$, and is a 2 -fold branched cover over some 6 -braid $\beta$ in $S^{2} \times S^{1}$. The hyperelliptic involution acts by isometry $\tau_{h}$ on the unit ball in the Thurston norm on $H_{2}(M, R)$; the class $[F]$ generated by the fibre spans the +1 -eigenspace of $\tau_{h}$. The orthogonal complement is the -1-eigenspace. It follows that $\tau_{h}\left[F^{\prime}\right]=-\left[F^{\prime}\right]$ for any genus 2 fibre $F^{\prime}$ not homologous to $F$ (from a different fibration). We may then assume, by Tollefson [To], that two parallel copies $F_{1}^{\prime}, F_{2}^{\prime}$ of $F^{\prime}$ are mapped to themselves by an orientation reversing involution. These surfaces project to disjoint surfaces $S_{1}, S_{2}$ in $S^{2} \times S^{1}$, with $\beta=\partial S_{1} \cup \partial S_{2}$. Moreover, each surface $S_{i}$ is a spine for a handlebody in a standard genus 2 Heegaard splitting of $S^{2} \times S^{1}$. This places serious restrictions on the possibilities for $\beta$, and asserts that the purported alternative fibration with fibre $F^{\prime}$ arises by Sakuma's construction [Sa]. Hence we call such a braid a Sakuma braid. 
At a more basic level, 6-braids induce permutations in the symmetric group $S_{6}$, which has order 720. For half of all possible permutations, any corresponding braid cannot have its component circles partitioned into two classes, each class being the boundary of either $S_{1}$ or $S_{2}$. For these cases, $F^{\prime}$ cannot exist. It is in this sense we assert 'most' fibrations are unique, in the statement of the theorem.

Hass [Has] has proved that if every element of a group $G$ acting freely on a 3-manifold $M$ preserves an element $\alpha \in H_{2}(M, Z)$ up to sign, then the quotient manifold $M / G$ is Haken (with incompressible surface arising from $\alpha$ ). This result applies whenever a manifold has a fibre which is 'unique' in any way forcing it to be homologically preserved by any group action.

Acknowledgment. The author would like to express his thanks to the organizers of the Hanoi Workshop for the opportunity to give the talk on which this paper is based, and to the many Vietnamese whose hospitality made the Workshop so enjoyable. In particular, he would like to thank Professors Yukio Matsumoto, Hyunh Mui and Mutsuo Oka for their kindness, patience and encouragement.

\section{REFERENCES}

[AR1] I.R. Aitchison and J.H. Rubinstein, 'An introduction to polyhedral metrics of non-positive curvature on 3-manifolds', in: Geometry of Low-Dimensional Manifolds: 2 (ed. S.K. Donaldson and C.B. Thomas), London Math. Soc. Lecture Notes 151 (Cambridge University Press, Cambridge, 1990), pp. 127-161.

[AR2] I.R. Aitchison and J.H. Rubinstein, 'Canonical surgery on alternating link diagrams', in: Knots 90 (ed. A. Kawauchi), (de Gruyter, Berlin, 1992), pp. 543-558.

[AR3] I.R. Aitchison and J.H. Rubinstein, 'Incompressible surfaces and the topology of 3-dimensional manifolds', J. Austral. Math. Soc. (Series A) 55 (1993), 1-22.

[ALR] I.R. Aitchison, E. Lumsden and J.H. Rubinstein, 'Cusp structure of alternating links', Invent. Math. 109 (1992), 473-494.

[Ca] A. Casson, 'On the Seifert fibred space conjecture', Talk at Kyoto, 1990.

[CLR] D. Cooper, D.D. Long and A.W. Reid, 'Bundles and finite foliations', preprint, 1993.

[Ga] D. Gabai, 'On 3-manifolds finitely covered by surface bundles', in: Low-Dimensional Topology and Kleinian Groups (ed. D.B.A. Epstein), London Math. Soc. Lecture Notes 112 (Cambridge University Press, Cambridge, 1986), pp. 145-155.

[Ga2] D. Gabai, 'Convergence groups are Fuchsian groups', preprint.

[GA] F. Gonzáles-Acuña, 'Dehn's construction on knots', Bol. Soc. Mat. Mexicana 15 (1970), 58-79.

[Has] J. Hass, 'Surfaces minimizing area in their homology class and group actions on 3-manifolds', Math. Zeit. 199 (1988), 501-509.

[HS] J. Hass and P. Scott, 'Homotopy equivalence and homeomorphism of 3-manifolds', Topology 31 (1992), 493-517.

[Hat] A. Hatcher, Communication via Hyam Rubinstein, Georgia, 1993.

[He1] J. Hempel, 3-Manifolds, Annals of Math. Studies 86 (Princeton University Press, Princeton, New Jersey, 1976).

[He2] J. Hempel, 'Orientation reversing involutions and the first Betti number for finite coverings of 3-manifolds', Invent. Math. 67 (1982), 133-142.

[Ja] W. Jaco, Lectures on three-manifold topology, Conf. Board of Math. Sci. 43 (American Math. Society, Providence, 1980).

[JS] W. Jaco and P. Shalen, Seifert-fibered Spaces in 3-Manifolds, Mem. Amer. Math. Soc. 220 
(1980).

[Joh] K. Johannson, Homotopy Equivalences of 3-manifolds with Boundary, Lecture Notes in Mathematics 761 (Springer-Verlag, Berlin 1979).

[Kn] H. Kneser, 'Geschlossene Flächen in dreidimensionalen Mannigfaltigkeiten', Jahresbericht der Deut. math. Verein 38 (1929), 248-260.

[Ko] S. Kojima, 'Isometry transformations of hyperbolic three-manifolds', Topology and its Appl. 29 (1988), 297-307.

[Li] W.B.R. Lickorish, 'A representation of orientable combinatorial 3-manifolds', Annals of Math. 76 (1962), 531-540.

[Lo] D. Long, 'Immersions and embeddings of totally geodesic surfaces', Bull. London Math. Soc. 19 (1987), 481-484.

[Lu] J. Luecke, 'Finite covers of 3-manifolds containing essential tori', Trans. Amer. Math. Soc. 310 (1988), 381-391.

[Ma] A.A. Markov, 'Unsolvability of the problem of homeomorphy', Proc. Int. Cong. Math. (1958), 300-306.

[Me] G. Mess, 'Centers of 3-manifold groups and groups which are coarse quasiisometric to planes', preprint.

[Mill] J. Millson, 'On the first Betti number of a constant negatively curved manifold', Annals of Math. 104 (1976), 235-247.

[Miln] J. Milnor, 'A unique factorisation theorem for 3-manifolds', Amer. J. Math. 84 (1962), 1-7.

[Moi] E. Moise, 'Affine structures in 3-manifolds $V$. The triangulation theorem and Hauptvermutung', Annals of Math. 56 (1952), 96-114.

[My] R. Myers, 'Open book decompositions of 3-manifolds', Proc. Amer. Math. Soc. 72 (1978), 397-402.

[Pa] C. Papakyriakopoulos, 'On Dehn's Lemma and the asphericity of knots', Annals of Math. 66 (1957), 1-26.

[Pen] R. Penner, 'A construction of pseudo-Anosov homeomorphisms', Trans. Amer. Math. Soc. 310 (1988), 179-197.

[Re] A. Reid, 'A non-Haken hyperbolic 3-manifold covered by a surface bundle', preprint (1992).

[Sa] M. Sakuma, 'Surface bundles over $S^{1}$ which are two-fold branched cyclic coverings of $S^{3}$, Math. Seminar Notes (Kobe) 9 (1981), 159-180.

[Sc] P. Scott, 'The geometries of 3-manifolds', Bull. London Math. Soc. 15 (1983), 401-487.

[Sc2] P. Scott, 'Strong annulus and torus theorems and the enclosing property of characteristic submanifolds of 3-manifolds', Quart. J. Math. Oxford (2) 35 (1984), 485-506.

[So1] T. Soma, 'Virtual fibers in hyperbolic 3-manifolds', Topology and its Appl. 41 (1991), $179-192$.

[So2] T. Soma, 'Virtual fibers in 3-manifold groups', J. London Math. Soc. (2) 43 (1991), 337-354.

[St] J. Stallings, 'On fibering certain 3-manifolds', in: Topology of 3-Manifolds (ed. M. Fort), (Prentice-Hall, 1962), pp. 95-100.

[Th1] W. Thurston, Lecture notes on the geometry and topology of 3-manifolds, (Princeton University, 1978).

[Th2] W. Thurston, 'Three dimensional manifolds, Kleinian groups and hyperbolic geometry', Bull. Amer. Math. Soc. 6 (1982), 357-381.

[Th3] W. Thurston, 'Hyperbolic structures on 3-manifolds II: Surface groups and 3-manifolds which fiber over the circle', unpublished preprint.

[Th4] W. Thurston, A norm for the homology of 3-manifolds, Memoirs of the Amer. Math. Soc. 339 (1986), 100-130.

[Th5] W. Thurston, 'On geometry and dynamics of diffeomorphisms of surfaces', Bull. Amer. Math. Soc. 19 (1988), 417-431.

[To] J. Tollefson, 'Periodic homeomorphisms of 3-manifolds fibered over $S^{1}$, Trans. Amer. Math. Soc. 223 (1976), 223-234. 
[Wa] F. Waldhausen, 'On irreducible 3-manifolds which are sufficiently large', Annals of Math. 87 (1968), 56-88.

[Wall] A. Wallace, 'Modifications and cobounding manifolds', Canad. J. Math. 12 (1960), 503-528.

Department of Mathematics

UNIVERSITY OF MELBOURNE

PARKVILle 3052, Victoria

Australia 